\title{
Gaussian Process Adaptive Incremental Backstepping Flight Control
}

\author{
Dmitry Ignatyev ${ }^{1}$, Hyo-Sang Shin ${ }^{2}$ and Antonios Tsourdos ${ }^{3}$ \\ Cranfield University, Cranfield MK43 OAL, UK
}

\begin{abstract}
The presence of uncertainties caused by unforeseen malfunctions in the actuation system or changes in aircraft behaviour could lead to aircraft loss of control during flight. The paper proposes almost model-independent control law combining recent developments in nonlinear control theory, data-driven methods, and sensor technologies by considering Gaussian Processes Adaptive augmentation for Incremental Backstepping control (IBKS) algorithm. IBKS uses angular accelerations and current control deflections to reduce the dependency on the aircraft model. However, it requires knowledge of control effectiveness. Conducted research shows that if the input-affine property of the IBKS is violated, e.g., in severe conditions with a combination of multiple failures, the IBKS can lose stability. Meanwhile, the GP-based estimator provides fast identification and the resultant GP-adaptive IBKS algorithm demonstrates improved stability and tracking performance. The performance of the algorithm is validated using a large transport aircraft flight dynamics model.
\end{abstract}

\section{Introduction}

During the last decades, aircraft safety significantly increased. Nevertheless, flight safety of passenger a viation in the presence of abnormalconditions, such as those caused by equipment failures and/or a dverse environmentalfactors, is an important problem. Analysis of accident and incidence reports revealed that the main contribution to the fatal accidents were due to aircraft Loss of Control In-Flight and Controlled Flight Into Terrain [1]. The main reasons caused these accidents are pilot mistakes, technical malfunctions, or their combination.

Recent developments in the areas of nonlinear control the ory, data-driven methods and sensor technology unveiled the way to the derivation of flight control laws that are significantly less dependent on nominalaircraft modeldata.

Recently, great efforts have been undertaken to develop a ircraft control design tools and techniques for analysis and improvement of safe flight [2-8]. The idea that non-conventional control strategies can prevent possible accidents and recover aircraft from dangerous situations stimulates researches toward fault-tolerant and adaptive flight control [9-12].

Gain-scheduling of linear feedback controllers is widely applied in commercial applications to achieve stabilization and satisfactory tracking performance of aircraft over a wide range of flight conditions $[13,14]$. In case of severe and unpredicted changing in aircraft behaviour such controllers cannot be used or can be used only with a restricted functionality.

Nonlinear Dynamics Inversion (NDI) and Backstepping (BS) techniques have become popular control strategies for adaptation since they can be used for global linearization of the system dynamics and controldecoupling [15-19]. The BS control has advantages in comparison with the NDI, namely, it is more flexible and it is based on Lyapunov stability theory. Later, to make the NDI and BS controls more robust and fault-tolerant an incremental-type sensorbased form has been proposed [20,21].

However, even in this formulation the controller still requires accurate knowledge of the control effectiveness, especially, if the system is not affine in control inputs because of non-linearity in actuators or due to large transport delays $[22,23]$. Additional a da ptation strategies a ugmenting the incremental-type controllers to reduce dependency on an a ircraft model were applied for a high-performance a ircraft model in Refs. [23,24]. Regardless of the fact that IBKS

\footnotetext{
${ }^{1}$ Senior Research Fellow, School of Aerospace, Transport and Manufacturing.

${ }^{2}$ Professor, School of Aerospace, Transport and Manufacturing, and AIAA Member Grade (if any) for second author.

${ }^{3}$ Head of Centre for Autonomous and Cyber-Physical Systems, School of Aerospace, Transport and Manufacturing, and AIAA Member Grade (if any)for third author.
} 
demonstrates robustness to some failures $[25,26]$ estimation of the control effectiveness improves fault-tolerant abilities of the system [22,27-30].

The most well-known and popular approach for on-line identification and implementation of in-direct adaptation is Recursive Least Squares (RLS) method [22,24,31,32]. However, RLS even with conventional Exponential Forgetting factor(EF) is not designed for tracking time-varying systems. Its convergence might be slow if the EF is close to one, whereas the error is large if the EF is small. Tuning Functions (TF) approach was proposed as natural expansion of the adaptive capabilities provided by the backstepping paradigm into identification of control efficiency $[17,22,24]$. This approach takes advantages of Lyapunov function design, which allows theoretical proof of stability to be obtained. However, these methods might be sensitive to measurement noise and thus additional supervising a lgorithms needs to be developed to switch-off the adaptation when signal-to-noise ratio is small. For example, a twolayer adaptive augmentation to IBKS control law was developed in Ref. [22].

Neural networks, including RadialBasis Functions (RBF), are quite popular for on -line identification and adaptive control since they are universal approximators and can match any uncertainty (for example, see. Refs. [33-35]). RBFs have advantage, namely, they are linear-in-the-parameters, as opposed to multilayer perceptron neural networks. However, performance of former approach is significantly determined by selection of the RBF centres. Normally, researches preallocate a fixed quantity of Gaussian RBF centres over the presumed domain $[33,36]$. The system states must stay close to the location of the preallocated RBF centres because a Gaussian RBF output decays exponentially away from its centre; otherwise, the system would not be able to capture the uncertainty.

To tackle the issues mentioned above we propose to use a Gaussian Process (GP) online identification framework to estimate aircraft control derivatives and perform in-direct adaptive control loop augmenting baseline IBKS controller. GP brings promising Bayesian paradigm to on-line identification and adaptive controlby considering the estimation as a statistical problem [37]. Within the proposed approach GPs utilize a Bayesian framework to represent uncertainties as a distribution over functions. It is assumed that the uncertainty and the model follow Gaussian distributions, with the uncertainty being estimated using its mean and covariance function. One of the advantages of the proposed method is that it does not require prior assumptions about operating domain. From the provided flight data, GP is able to dynamically choose new kernel loca tions to guarantee domain coverage. Furthermore, mea surement noise is explicitly handled, and parameters such as the centres of RBFs does not require pre-allocation. GP approach allows Bayesian inference to overcome shortcomings of the standard gradient based parameter update laws, e.g. lack of convergence guarantees and possible instabilities under noise presence [31,38]. This method was applied fordesign of direct adaptive control GP-MRAC in Refs. [39,40], where GP was used to match uncertainty to produce compensating control commands. In the current paper, unlike GP-MRAC, we propose to develop a GP-based estimation of control efficiency and then the updated values are fed to the baseline IBKS controller, which performs control of the aircraft. We propose a budgeted sparse GP algorithm suitable for on-line identification and adaptation. The developed approach is compared with EF RLS and TF in three different scenarios, when uncertainties are introduced in the control efficiency.

The present paper demonstrates results from the European project INCEPTION, which was seeking the development of fault-tolerant Automatic Flight Control System for fixed-wing aircraft allying incremental control strategies, adaptive augmentation and envelope protection [41]. The proposed augmentation is designed to improve stability and tracking performance of the IBKS baseline controller by providing actual information about control effectiveness in case of uncertainty or failure.

The paper is organized in the following way. A very brief overview of the flight dynamics and IBKS control strategy are given in Sections 2 and 3 correspondingly. Section 4 provides a description of the identification framework in general. The budgeted sparse GP algorithm is considered in Sections 5. EF RLS and TF estimation algorithms are presented in Section 6. Section 7 deals with a simulation results of the proposed framework. Finally, concluding remarks are summarized in Section 8.

\section{Flight Dynamics Model}

Equations of motions of the aircraft can be represented with kinematics and dynamics models. The kinematics of the aircraft is described by the attitude state vector $\xi=\left[\begin{array}{lll}\phi & \theta\end{array}\right]^{T}$, where $\phi, \theta, \beta$ are roll, pitch and sideslip angles.

$$
\dot{\xi}=\mathbf{f}_{\xi}+T_{\xi} \mathbf{\omega}
$$

where 


$$
\begin{gathered}
\mathbf{f}_{\xi}=\left[\begin{array}{llll}
0 & 0 & -\frac{A_{x}}{V_{t}} \cos \alpha \sin \beta+\frac{A_{y}}{V_{t}} \cos \beta-\frac{A_{z}}{V_{t}} \sin \alpha \sin \beta+\frac{g_{y}}{V_{t}}
\end{array}\right]^{T} \\
T_{\xi}=\left[\begin{array}{ccc}
1 & \sin \phi \tan \theta & \cos \phi \tan \theta \\
0 & \cos \phi & -\sin \phi \\
\sin \alpha & 0 & -\cos \alpha
\end{array}\right]
\end{gathered}
$$

$\boldsymbol{\omega}=[p, q, r]^{T}$ is the rotational rate vector. Specific forces $A_{x}, A_{y}$ and $A_{z}$ can be directly measured by the accelerometers. $V_{t}$ is the true airspeed, $\alpha$ is the angle of attack, $g_{y}$ is the y-axis component of the gravitational acceleration calculated in the wind reference frame.

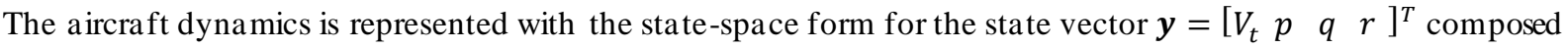
of airspeed $V_{t}$, roll rate $p$, pitch rate $q$ and yaw rate $r$ :

$$
\dot{\mathbf{y}}=\mathbf{f}_{y}(\mathbf{y}, \mathbf{u})
$$

Control inputs $\boldsymbol{u}=\left[\delta_{\text {ail }_{l L}} \delta_{\text {ail }_{l R}} \delta_{\text {ail }_{O L}} \delta_{\text {ail }_{O R}} \delta_{e_{I L}} \delta_{e_{I R}} \delta_{e_{O L}} \delta_{e_{O R}} \delta_{r_{u}} \delta_{r_{l}} \delta_{T_{I L}} \delta_{T_{I R}} \delta_{T_{O L}} \delta_{T_{O R}}\right]^{T}$ are the inner-left, innerright, outer-left, and outer-right ailerons; inner-left, inner-right, outer-left, and outer-right elevators; upper and lower rudders; outer-left, inner-left, inner-right, and outer-right engines throttle.

The nonlinear dynamics $\boldsymbol{f}_{y}(\boldsymbol{y}, \boldsymbol{u})$ is linearized using the incremental dynamics approach for the incremental controller design and thus the precise description is not provided here.

\section{Incremental Backstepping}

Sensor-based technique utilizing Incremental Dynamics (ID) applied to obtain an IBKS controller, which is less dependent on the system model, is discussed in Refs. [42,43]. Below, we will just follow a brief description of this controller. Details could be found in the original papers. IBKS computes incremental commands employing acceleration feedback estimations to extract unmodeled flight dynamics. In the present study, we are using this controller as a baseline controller, which is augmented with the proposed budgeted sparse GP parameter estimator.

\section{A. Incremental Dynamics Model}

It is assumed here that the system dynamics is described by the following nonlinear equations:

$$
\dot{\mathbf{x}}=\mathbf{f}_{\mathbf{x}}(\mathbf{x}, \mathbf{u})
$$

where $\boldsymbol{f}_{\boldsymbol{x}}: \mathbb{R}^{n} \times[0, \infty) \rightarrow \mathbb{R}^{n}$ is Lipschitz continuous function, $\mathbf{x}$ and $\boldsymbol{u}$ are the state and the control input vectors. Expanding Eq. (3) into the Taylor series around $\left(\boldsymbol{x}_{0}, \boldsymbol{u}_{0}\right)$ corresponding to the previous time moment $t_{0}$ the dynamics (3) can be expressed in the following form

$$
\dot{\mathbf{x}} \cong \dot{\mathbf{x}}_{0}+\frac{\partial \mathbf{f}_{\mathbf{x}}(\mathbf{x}, \mathbf{u})}{\partial \mathbf{x}}\left(\mathbf{x}-\mathbf{x}_{0}\right)+\frac{\partial \mathbf{f}_{\mathbf{x}}(\mathbf{x}, \mathbf{u})}{\partial \mathbf{u}}\left(\mathbf{u}-\mathbf{u}_{0}\right)
$$

Assuming that the increment in state $\Delta \boldsymbol{x}=\boldsymbol{x}-\boldsymbol{x}_{0}$ is much smaller than the increment in both state derivative $\Delta \dot{\boldsymbol{x}}=\dot{\boldsymbol{x}}-\dot{\boldsymbol{x}}_{0}$ and input $\Delta \boldsymbol{u}=\boldsymbol{u}-\boldsymbol{u}_{0}$, the dynamics (4) can be further simplified

$$
\Delta \dot{\mathbf{x}} \cong B_{0} \Delta \mathbf{u}
$$

where $B_{0}=\frac{\partial \boldsymbol{f}_{\boldsymbol{x}}(\boldsymbol{x} \boldsymbol{u})}{\partial \boldsymbol{u}}$ is a control effectiveness matrix.

The dynamics equation in the form (5) states that the ID of the system is produced by the control input increment. For the implementation of such a concept, it is assumed that sampling time is small. In this case, the assumption that $\Delta \boldsymbol{x} \ll \Delta \dot{\boldsymbol{x}}$ and $\Delta \boldsymbol{x} \ll \Delta \boldsymbol{u}$ becomes possible for a real aircraft because the control surface deflections directly affect the angular accelerations, whereas the angular rates are only changed by integrating these angular accelerations. Actuators 
are assumed to be instantaneously fast such that the demanded input increment can be achieved within the small sampling time. In addition, the sensors are assumed to be ideal, i.e. providing state derivative without errors.

\section{B. Attitude Controller}

The ID idea combined with the backstepping paradigm was utilized to design the aircraft baseline controller $[42,43]$. To increase the control robustness and simplify its implementation, both angle and rate controllers using ID were formulated. The general structure of the baseline controller with the revealed interaction between attitude and rate controllers is provided in Fig.1.

Equations (1) and (2) constitute the system dynamics. Introducing the kinematics tracking error variable $\boldsymbol{z}_{\xi}=\xi_{d}-\xi$, where $\xi_{d}$ is the desired kinematics state vector, the sub-system (1) can be reformulated in terms of $\boldsymbol{z}_{\xi}$ :

$$
\dot{\mathbf{z}}_{\xi}=\dot{\xi}_{d}-\mathbf{f}_{\xi}-T_{\xi} \mathbf{\omega}
$$

The general idea behind the backstepping is to consider the state vector $\boldsymbol{\omega}=\left[\begin{array}{lll}p & q & r\end{array}\right]^{T}$ from (2) as a control input for $\boldsymbol{z}_{\xi}$ subsystem (6). Since $\boldsymbol{\omega}$ is just a state variable and not the real control input, it is called a virtual control input.

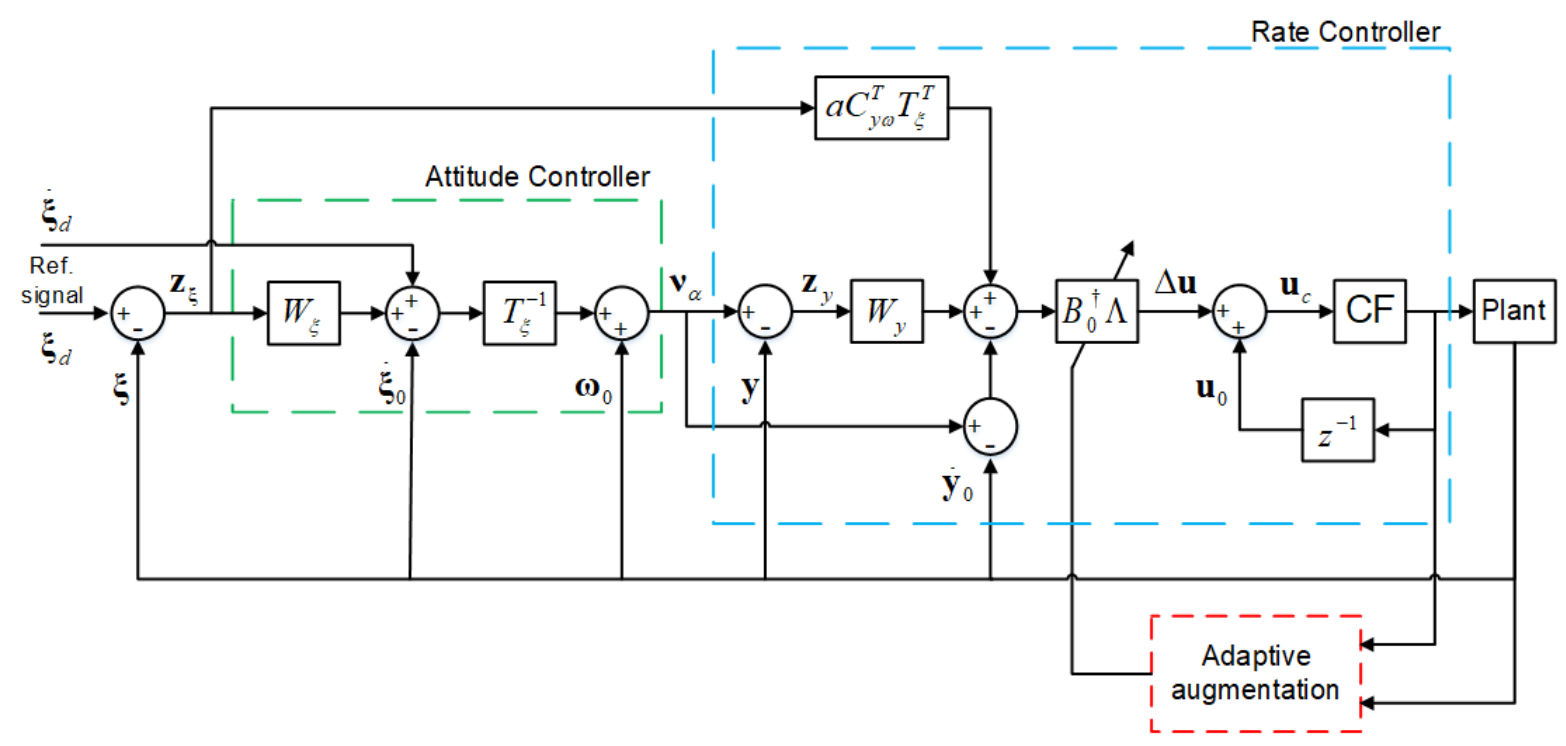

Fig.1 Controller structure

For the $\boldsymbol{z}_{\xi}$ subsystem a Candidate Lyapunov Function (CLF) $V_{\xi}$ is selected:

$$
V_{\xi}=\frac{1}{2} \mathbf{z}_{\xi}^{T} \mathbf{z}_{\xi},
$$

which is positive for the whole domain, excluding the origin, where it equals to zero.

For the asymptotic convergence of the error, the CLF derivative must be strictly negative along the solutions of (6). Considering a positive definite matrix $W_{\xi} \in \mathbb{R}^{3 \times 3}$, the CLF is strictly negative if:

$$
\dot{V}_{\xi}=\mathbf{z}_{\xi}^{T} \dot{\mathbf{z}}_{\xi}=-\mathbf{z}_{\xi}^{T} W_{\xi} \mathbf{z}_{\xi}
$$

The kinematics tracking error dynamics can be represented in the incrementalform

$$
\dot{\mathbf{z}}_{\xi}=\dot{\xi}_{d}-\dot{\xi}_{0}-T_{\xi}\left(\omega-\omega_{0}\right)
$$

Substituting the expression $\dot{\boldsymbol{z}}_{\xi}=-W_{\xi} \mathbf{z}_{\xi}$ derived from (8), the following tracking error dynamics is obtained 


$$
\dot{\xi}_{d}-\dot{\xi}_{0}-T_{\xi}\left(\omega-\omega_{0}\right)+W_{\xi} \mathbf{z}_{\xi}=0
$$

The virtual control law $v_{\alpha}=\boldsymbol{\omega}$ can be obtained by inversion of (10) with respect to $\boldsymbol{\omega}$

$$
v_{\alpha}=\boldsymbol{\omega}_{0}+T_{\xi}^{-1}\left(W_{\xi} \mathbf{z}_{\xi}+\dot{\xi}_{d}-\dot{\xi}_{0}\right)
$$

since $T_{\xi}$ is invertible for the transport aircraft within the flight envelope. The control law (11) is used as a desired value for the virtual control input $\boldsymbol{\omega}$.

\section{Rate Controller}

The difference between the dynamics of the state variable $\boldsymbol{y}=\left[V_{t} \boldsymbol{\omega}^{T}\right]^{T}$ and its desired value $\boldsymbol{y}_{d}=\left[V_{t d} \quad \boldsymbol{\omega}_{d}^{T}\right]^{T}$ is defined as the dynamics tracking error variable $\boldsymbol{z}_{\boldsymbol{y}}=\boldsymbol{y}_{d}-\boldsymbol{y}$. It should be noted that the airspeed is introduced as a state to the dynamics state vector in order to design the controller that simultaneously tracks the airspeed and angular rates of the aircraft. To design a control law $\mathbf{u}$ that ensures that $\boldsymbol{z}_{y}$ converges to zero, the following CLF for the complete $\left(\mathbf{z}_{\xi}, \boldsymbol{z}_{y}\right)$-system is formed:

$$
V_{y}=V_{\xi}+\frac{1}{2 a} \mathbf{z}_{y}^{T} \mathbf{z}_{y}
$$

where $a$ is a design scale factor. Similar to design of CLF for the $z_{\xi}$ subsystem in (8), here a matrix $W_{y} \in \mathbb{R}^{4 \times 4}$ is assumed to be a positive definite matrix such that

$$
\dot{V}_{y}=\dot{V}_{\xi}+\frac{1}{a} \mathbf{z}_{y}^{T} \dot{\mathbf{z}}_{y}=-\mathbf{z}_{\xi}^{T} W_{\xi} \mathbf{z}_{\xi}-\frac{1}{a} \mathbf{z}_{y}^{T} W_{y} \mathbf{z}_{y},
$$

Thus, the error $\boldsymbol{z}_{y}$ converges a symptotically to zero since the derivative of the CLF $V_{y}$ is strictly negative for nonzero errors. The tracking error dynamics in the incremental representation has the following form:

$$
\dot{\mathbf{z}}_{y}=\dot{\mathbf{y}}_{d}-\dot{\mathbf{y}}_{0}-B_{0}\left(\mathbf{u}-\mathbf{u}_{0}\right)
$$

The selection matrix $C_{y \omega}=\left[\begin{array}{ll}\mathbf{0}_{3} & I_{3}\end{array}\right]$, which performs the mapping $\boldsymbol{\omega}=C_{y \omega} \boldsymbol{y}$, is introduced. Combining the incrementaldynamics of $\boldsymbol{z}_{\xi}(9)$ and $\boldsymbol{z}_{y}(14)$, one can obtain

$$
\boldsymbol{z}_{\xi}^{T}\left(\dot{\boldsymbol{\xi}}_{d}-\dot{\boldsymbol{\xi}}_{0}-T_{\xi}\left(\boldsymbol{v}_{\alpha}-C_{y \omega} \boldsymbol{z}_{y}-\boldsymbol{\omega}_{0}\right)+W_{\xi} \mathbf{z}_{\xi}\right)+\frac{1}{a} \boldsymbol{z}_{y}^{T}\left(\dot{\boldsymbol{y}}_{d}-\dot{\boldsymbol{y}}_{0}-B_{0}\left(\boldsymbol{u}-\boldsymbol{u}_{0}\right)+W_{y} \mathbf{z}_{y}\right)=0 .
$$

Eventually, for non-zero errors, substituting (11) into (15) and solving it with respect to $\mathbf{u}$, the resultant control law is designed

$$
\mathbf{u}_{c}=\mathbf{u}_{0}+B_{0}^{-1} \Lambda\left(a C_{y \omega}^{T} T_{\xi}^{T} \mathbf{z}_{\xi}+W_{y}\left(\mathbf{y}_{d}-\mathbf{y}\right)+\dot{\mathbf{y}}_{d}-\dot{\mathbf{y}}_{0}\right)
$$

To attenuate the measurement noise and increase the control robustness, $B_{0}$ is multiplied by a diagonalmatrix $\Lambda>$ 0 with elements $\lambda_{i i} \in[0,1]$.

The control law in the form (16) requires inversion of the matrix $B_{0}$, which is not square for the overactuated modern transport aircraft. To tackle this issue, Moore-Penrose Pseudo-inverse is applied [43], and

$$
B_{0}^{\dagger}=B_{0}^{T}\left(B_{0} B_{0}^{T}\right)^{-1}
$$

is used in (16) instead of $B_{0}$. 


\section{Command Filter}

To a void infea sible commands provided by the controller, a Command Filter (CF) is added to the controller output. For the incremental controllers, the $\mathrm{CF}$ is used to constrain the input to respect the actuators dynamics and saturation.

Taking into account the influence of the CF $\chi \in \mathbb{R}^{3}$ on the tracking error $\boldsymbol{z}_{y}$ (14) the dynamics of the modifies tracking error $\overline{\boldsymbol{z}}_{y}$ is introduced [24]

$$
\dot{\mathbf{z}}_{y}=\dot{\mathbf{y}}_{d}-\dot{\mathbf{y}}_{0}-B_{0}\left(\mathbf{u}-\mathbf{u}_{0}\right)-\dot{\boldsymbol{\chi}}
$$

Effect of the CF on the tracking error can be estimated by the stable linear filter [24]:

$$
\dot{\chi}=-W_{y} \chi+B_{0}\left(\mathbf{u}_{C F}-\mathbf{u}_{c}\right),
$$

where $\boldsymbol{u}_{C F}$ is the controller output afterCF.

\section{On-Line Estimation of Control Effectiveness}

Finally, the cascaded baseline controller consists of attitude and rate controllers (11) and (16). Both attitude and rate controllers have the similar control structure, namely, the control signal compensating the difference b etween the reference and mea sured (or estimated) state variables is added to the current value of the control input. Such a structure is very simple and robust to possible uncertainties. However, the precise knowledge of the control input matrices $T_{\xi}$ and $B_{0}$ is required for stable performance of the algorithm. The precise value of $T_{\xi}$ within the flight envelope can be easily determined because it represents the kinematic relationships. The matrix $B_{0}$ specifies the control effectiveness, which might change during flight because of changing of environmentalconditions, structural deformations, failures etc. Hence, an unmodeled actuator dynamics is a source of uncertainty.

The main purpose of the adaptive augmentation for the IBKS is to compensate the effect of these uncertainties and to improve performance and stability of the IBKS controller. This paper introduces a GP online identification framework for estimating the aircraft control derivatives contained in matrix $B_{0}$. Interaction of the adaptive augmentation with the baseline controller is demonstrated in Fig.1. The adaptive augmentation block performs indirect adaptation by online estimation and adjustment of the control effectiveness matrix $B_{0}$.

An aircraft flight control system sends the same signals for all individual control surfaces, making the individual signals are proportional to each other and causing a high-correlation between the individual signals. If all the input signal forms look the same, then any a lgorithm trying to assign values for the control effectiveness of each individual control will fail, because it is impossible to determine which of the multiple inputs, moved in the same manner, was responsible for changes in the aerodynamic forces and moments. Input forms that are completely decorrelated will give the most accurate control effectiveness estimates. Unfortunately, when a feedback control system is operating, desired input forms become distorted by the feedback control. To tackle this issue, we use a priori information through fixing the effectiveness of all but one of the correlated control surfaces to a priori values [32].

Furthermore, while identifying the effectiveness of a certain control surface, the aircraft is demanded to perform manoeuvres with reduced coefficients in the allocation matrix $W_{s} D_{u}$ for all control effectors responsible for this motion, except the coefficient relating to the control surface under study. In such a ca se, the control signal is split into two signals, the first one is for the control surface of which effectiveness is treated, while the second signal is for all other surfaces from the pool. Thus, the first signal is responsible for generating the required information for identification and second one is used for guaranteeing the aircraft stability.

\section{Gaussian Processes On-Line Identification and Adaptive Augmentation}

GP utilizes Bayesian paradigm for on-line identification of control efficiency and the adaptive control by considering the identification as a statistical problem [37]. GP is non-parametric because the "parameters" to be identified are functions $f_{x}$ of an input variable $x \in R^{d}$. Function $f$ is characterised by its statistics, namely, by the mean $\langle f\rangle$ and the covariance, which is also called the kernel $K_{0}\left(x, x^{\prime}\right)=\operatorname{Cov}\left(\varsigma, \varsigma^{\prime}\right)$ [44]. The a priori assumption is that $f$ is a Gaussian process. Indeed, according to the Central Limit Theorem any sufficiently large set of random samples $f_{i}$ is considered to have normal distribution. Within the Bayesian framework, given a set of input-output observations $\left(x_{n}, S_{n}\right)(n=1, \ldots, N)$ the posterior distribution of the process $f_{x}$ is computed via prior and the likelihood. 
Csató and Opper [37] proposed a representation of posterior means $\left\langle f_{x}\right\rangle_{t}=\left\langle f\left(x_{t}\right)\right\rangle_{t}$ and the posterior covariance $K_{t}\left(x, x^{\prime}\right)$, where $t$ denotes the number of data points, with a finite linear combinations of kernels $K_{0}\left(x, x_{i}\right)$ evaluated at the training inputs $x_{i}$. Using sequential projections of the posterior process on the manifold of Gaussian processes, approximate recursions for the effective parameters of these representations can be obtained. To avoid enormous growth of the size of representations the elegant algorithm for extraction of a smaller subset of input data is proposed. Such a subsets allows an on-line sparse representation of the posterior process, which is used to predict the GP model.

The posterior expectations within the Bayesian approach are conventionally expressed by high-dimensional integrals. Obviously, this is not applicable for on-line identification. However, it was shown in [37] that the posterior mean and the posterior covariance of the process arbitrary inputs can be expressed as a combination of a finite set of parameters which depend on the training data only. To make Bayesian interference trackable on -line, the posterior is projected to the closest Gaussian process by a single sequentialsweep through the examples.

The posterior GP approximation with its posterior means and the posterior covariance can be estimated using the initial kernel $K_{0}\left(x, x^{\prime}\right)$ and the likelihoods:

$$
\begin{gathered}
\left\langle f_{x}\right\rangle_{t}=\boldsymbol{\alpha}_{t}^{T} \boldsymbol{k}_{x} \\
K_{t}\left(x, x^{\prime}\right)=K_{0}\left(x, x^{\prime}\right)+\boldsymbol{k}_{x}^{T} \boldsymbol{C}_{t} \boldsymbol{k}_{x^{\prime}}
\end{gathered}
$$

where $\boldsymbol{k}_{x}=\left[K_{0}\left(x_{1}, x\right), \ldots, K_{0}\left(x_{t}, x\right)\right]^{T}$ is the kernel functions, $\boldsymbol{\alpha}_{t}=\left[\alpha_{t}(1), \ldots, \alpha_{t}(t)\right]^{T}$ is the coefficient vector, $\boldsymbol{C}_{t}=$ $\left\{C_{t}(i j)\right\}_{i, j=1, t}$. is the coefficient matrix. It should be noted that coefficients $\alpha_{t}(i)$ and $C_{t}(i j)$ do not depend on $x$ and $x^{\prime}$ [37]. For the regression problems, Radial Basis Functions (RBF) are quite popular choice for kernel functions $[4,37,39,40]$

$$
K\left(x, x^{\prime}\right)=\exp \left(\frac{\left\|x-x^{\prime \prime}\right\|^{2}}{2 \sigma_{x}^{2}}\right)
$$

\section{A. Online Learning}

The recursive update of the GP parameters in Eq. (20) can be performed via the following equations:

$$
\begin{gathered}
\boldsymbol{\alpha}_{t+1}=T_{t+1}\left(\boldsymbol{\alpha}_{t}\right)+q^{(t+1)} \boldsymbol{s}_{t+1}, \\
\boldsymbol{C}_{t}=U_{t+1}\left(\boldsymbol{C}_{t}\right)+r^{(t+1)} \boldsymbol{s}_{t+1} \boldsymbol{s}_{t+1}^{T}, \\
\boldsymbol{s}_{t+1}=T_{t+1}\left(\boldsymbol{C}_{t} \boldsymbol{k}_{t+1}\right)+\boldsymbol{e}_{t+1}
\end{gathered}
$$

where $\boldsymbol{k}_{t+1}=\boldsymbol{k}_{x_{t+1}}$ and $\boldsymbol{e}_{t+1}$ the $t+1$-th unit vector and $\boldsymbol{s}_{t+1}$ is introduced for clarity. Operators $T_{t+1}$ and $U_{t+1}$ extend a $t$-dimensional vector and matrix to a $t+1$-dimensional one by appending zeros at the end of the vector and to the last row and column of the matrix respectively.

For the RBF kernel functions the $q^{(t+1)}$ and $r^{(t+1)}$ are defined as follows

$$
\begin{gathered}
q^{(t+1)}=\left(\varsigma-\boldsymbol{\alpha}_{t}^{T} \boldsymbol{k}_{x}\right) / \sigma_{x}^{2} \\
r^{(t+1)}=-1 / \sigma_{x}^{2}
\end{gathered}
$$

where $\sigma_{x}^{2}=\sigma_{0}^{2}+\boldsymbol{k}_{x}^{T} \boldsymbol{C}_{t} \boldsymbol{k}_{x}+k_{x}^{*}, k_{x}^{*}=K_{0}(x, x)$. One can conclude that the dimension of the vector $\boldsymbol{\alpha}$ and the size of matrix $\boldsymbol{C}$ increases with each data point added since $\boldsymbol{e}_{t+1}$ is the $t+1$-th unit vector.

The updates in the form of Eqs. (22) has a drawback since the number of parameters increa ses quadratically with the number of training examples. An effective way of controlling the number of parameters was proposed in Ref. [37], namely, sparseness within the GP framework was introduced. According to this approach, the update of the GP parameters is implemented without increase in the number of parameters $\boldsymbol{\alpha}$ and $\boldsymbol{C}$ when, according a certain criterion, the error due to the approximation is not too large.

If the new input $x_{t+1}$ is such that

$$
\boldsymbol{K}_{\mathbf{0}}\left(x, x_{t+1}\right)=\sum_{i=1}^{t} \hat{e}_{t+1}(i) K_{0}\left(x, x_{i}\right)
$$


is true for all $x$, then the update can be achieved exactly. In this case, the updated process in the form Eq. (20) is represented by only the first $t$ inputs, but with "renorma lised" para meters $\widehat{\boldsymbol{\alpha}}$ and $\widehat{\boldsymbol{C}}$ and the update(22) is implemented without extending the size of the parameters $\boldsymbol{\alpha}$ and $\boldsymbol{C}$ and $\boldsymbol{s}_{t+1}$ as follows:

$$
\begin{gathered}
\widehat{\boldsymbol{\alpha}}_{t+1}=\boldsymbol{\alpha}_{t}+q^{(t+1)} \hat{\boldsymbol{s}}_{t+1}, \\
\widehat{\boldsymbol{\boldsymbol { C }}}_{t}=\boldsymbol{C}_{t}+r^{(t+1)} \widehat{\boldsymbol{s}}_{t+1} \widehat{\boldsymbol{s}}_{t+1}^{T}, \\
\hat{\boldsymbol{s}}_{t+1}=\boldsymbol{C}_{t} \boldsymbol{k}_{t+1}+\hat{\boldsymbol{e}}_{t+1}
\end{gathered}
$$

where $\widehat{\boldsymbol{\alpha}}_{t+1}, \widehat{\boldsymbol{C}}_{t}$ and $\widehat{\boldsymbol{s}}_{t+1}$ are $t$-th unit vectors.

Obviously, for most kernels and inputs $x_{t+1}$ relationship (24) does not hold for all input x. However, the updates in the form of (25) might be used for approximations if $\hat{\boldsymbol{e}}_{t+1}$ is determined by minimising the error measure

$$
\left\|K_{0}\left(\cdot, x_{t+1}\right)-\sum_{i=1}^{t} \hat{e}_{t+1}(i) K_{0}\left(\cdot, x_{i}\right)\right\|^{2},
$$

where $\|\cdot\|$ is a norm in a space of functions of inputs $x$. If the norm is defined via the inner product of the reproducing kernel Hilbert space (RKHS) generated by the kernel $K_{0}$, then minimising (26), one can obtain the following expression

$$
\hat{\boldsymbol{e}}_{t+1}=\boldsymbol{K}_{t}^{-1} \boldsymbol{k}_{t+1},
$$

where $\boldsymbol{K}_{t}=\left\{K_{0}\left(x_{i}, x_{j}\right)\right\}_{i, j=1, t}$ is the Gram matrix. In this case, the equation

$$
\widehat{K}_{0}\left(x, x_{t+1}\right)=\sum_{i=1}^{t} \hat{e}_{t+1}(i) K_{0}\left(\cdot, x_{i}\right)
$$

gives the orthogonalprojection of the function $K_{0}\left(x, x_{t+1}\right)$ on the linear span of the functions $K_{0}\left(x, x_{i}\right)$.

The update rule (25) is performed when a measure of the approximation error

$$
\gamma_{t+1}=k_{t+1}^{*}-\boldsymbol{k}_{t+1}^{T} \boldsymbol{K}_{t}^{-1} \boldsymbol{k}_{t+1}
$$

does not exceed some tolerance level $\epsilon_{t o l}>0$. Here, $k_{t+1}^{*}=K_{0}\left(x_{t+1}, x_{t+1}\right)$. The Eq. (29) has a geometrical interpretation, namely, it is a square norm of the "residual vector" from the projection in the RKHS. Alternatively, it measures the "novelty" of the current input. If $\gamma_{t+1}$ is higher than a threshold value then the current input holds additional information as compared to the existing set of inputs, which is called "basis vector set" or $B V$ set, and thus it should be added to this set. Proceeding sequentially, some of the inputs are left out and others are included in $B V$ set. However, because of the projection (28) the inputs left out from $B V$ set will still contribute to the final GP configuration - the one used for prediction and to measure the posterior uncertainties. But the latter inputs will not be stored and do not lead to an increase of the size of the parameter set [37].

To avoid computationally expensive inversion of the Gram matrix the recursive calculation of the inverse Gram matrix can be employed [37]:

$$
Q_{t+1}=Q_{t}+\gamma^{-1}\left(\hat{\boldsymbol{e}}_{t+1}-\boldsymbol{e}_{t+1}\right)\left(\hat{\boldsymbol{e}}_{t+1}-\boldsymbol{e}_{t+1}\right)^{T},
$$

where $\boldsymbol{e}_{t+1}$ is the $t+1$-th unit vector. All matrix inversion is excluded using this recursion relationship. The Gram matrix is guaranteed to be non-singular since only inputs with novel information about Gau ssian process are included in the $B V$ set and $\gamma_{t+1}>0$ guarantees non-singularity of the extended Gram matrix.

\section{B. Deleting a Basis Vector}

Recursive update of the GP parameters (22) is implemented while the $B V$ set does not exceed the budget, namely, the maximum number of elements in $B V$. Thus, a pruning procedure should be introduced. When a new example is estimated as novel, this procedure should get rid of one of the basis vectors and replace it by the new input vector. Two different strategies can be applied for selection of the vector from the $B V$ set. The first strategy supposed to add a novel input vector instead of the oldest basis vector [40]. The second strategy [37] proposes to replace the basis vector with the smallest error. The former might be preferred for a fast-varying process. However, here we will follow the later approach since it provides enhanced richness of the $B V$ set. 
The removal procedure assumes that the respective $B V$ was added and the previous update step (25) was implemented. In this case $\boldsymbol{\alpha}_{t+1}$ has $t+1$ elements, and $C_{t+1}$ and $Q_{t+1}$ are the $(t+1) \times(t+1)$ matrices. If we assume that the last added element should be deleted the decomposition of the $\boldsymbol{\alpha}_{t+1}, C_{t+1}$ and $Q_{t+1}$ could be represented as follows:

$$
\boldsymbol{\alpha}_{t+1}=\left[\begin{array}{c}
\boldsymbol{\alpha}_{\boldsymbol{t}}^{\boldsymbol{t}} \\
\alpha^{r}
\end{array}\right], C_{t+1}=\left[\begin{array}{cc}
C_{t}^{l} & \boldsymbol{c}^{r} \\
\boldsymbol{c}^{r T} & c^{r}
\end{array}\right], Q_{t+1}=\left[\begin{array}{cc}
Q_{t}^{l} & \boldsymbol{q}^{r} \\
\boldsymbol{q}^{r T} & q^{r}
\end{array}\right]
$$

where $C_{t}^{l}$ and $Q_{t}^{l}$ are $t \times t$ sub-matrices extracted from the $(t+1) \times(t+1)$ matrices $C_{t+1}$ and $Q_{t+1}$. For the sake of simplicity, this representation is shown for the case when the last element should be removed, however, similar partitioning could be done for a general case. Updating equations for the element deleting case are the following:

$$
\begin{aligned}
& \widehat{\boldsymbol{\alpha}}=\boldsymbol{\alpha}_{\boldsymbol{t}}^{\boldsymbol{l}}-\alpha^{r} \frac{\boldsymbol{q}^{r}}{q^{r}} \\
& \hat{C}=C_{t}^{l}+c^{r} \frac{\boldsymbol{q}^{r} \boldsymbol{q}^{r T}}{q^{r 2}}-\frac{1}{q^{r}}\left[\boldsymbol{q}^{r} \boldsymbol{c}^{r T}+\boldsymbol{c}^{r} \boldsymbol{q}^{r T}\right], \\
& \hat{Q}=Q_{t}^{l}-\frac{\boldsymbol{q}^{r} \boldsymbol{q}^{r T}}{q^{r}},
\end{aligned}
$$

where $\widehat{\boldsymbol{\alpha}}, \hat{C}$ and $\widehat{Q}$ are the parameters after the deletion of the last basis vector and $\boldsymbol{\alpha}_{\boldsymbol{t}}^{\boldsymbol{l}}, C_{t}^{l}, Q_{t}^{l}, \alpha^{r}, \boldsymbol{c}^{r}, \boldsymbol{q}^{r}, c^{r}$ and $q^{r}$ are taken from GP parameters before deletion.

To decide the element of the $B V$ set to be deleted a score measure for each element $i$ is calculated

$$
\varepsilon_{i}=\frac{\left|\alpha_{t+1}(i)\right|}{Q_{t+1}(i, i)}
$$

The basis vector with minimal score (33) is deleted. This method provides deleting of a basis vector from the $B V$ set with minimal loss of information. Finally, the budgeted sparse GP algorithm is summarized by Algorithm 1.

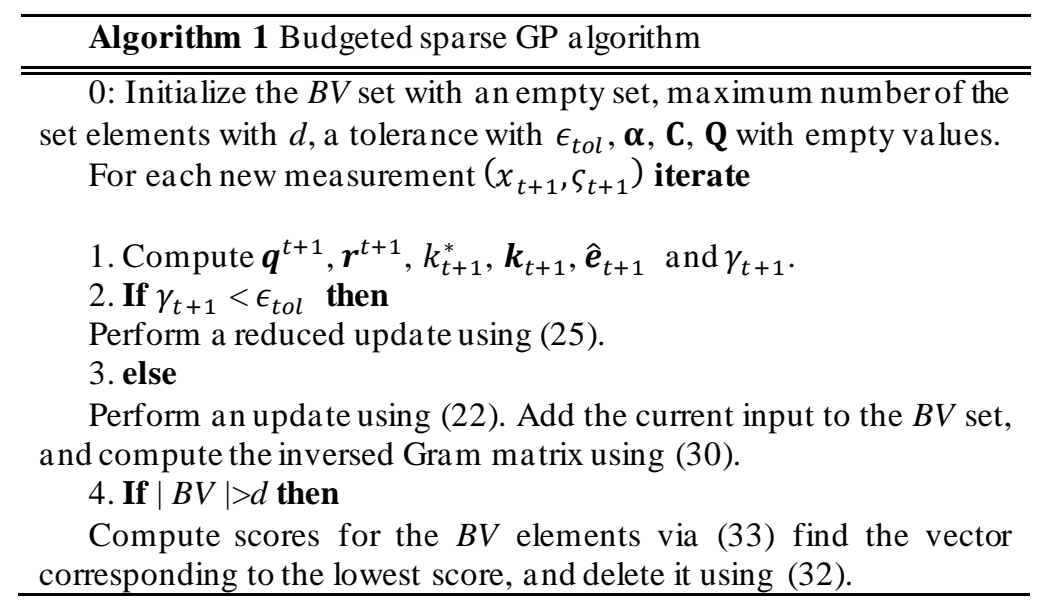

\section{Control effectiveness estimation and IBKS adaptation loop}

Within the proposed framework the control efficiency is approximated via GP, which is characterized with its mean $\left\langle f_{x}\right\rangle$ and covariance $K\left(x, x^{\prime}\right)$. For smooth identification process the component of the input vector are normalized. The output observations are the instanta neous controlefficiency values $\varsigma$ estimated from (5) by dividing the increment of state derivate $\Delta \dot{x}$ by increment of control input $\Delta u$

$$
\varsigma_{i}=\frac{\Delta \dot{x}}{\Delta u}
$$

To a void singularity of the estimation due to division in (34), we added additional check of the input data, na mely, 


$$
\Delta u<\epsilon_{u_{t o l}} .
$$

Further, the estimated value of the control efficiency is supplied to the IBKS controller as a new value of a corresponding coefficient in $B_{0}$.

\section{Incremental Backstepping}

We compared the proposed GP augmentation for IBKS with other adaptive strategies based on Recursive Least Square with exponentialforgetting and Tuning functions.

\section{A. Recursive Least Square with Exponential Forgetting}

The effectiveness is estimated online using the Recursive Least Square (RLS) with exponential forgetting (EF), which is commonly used for the real-time system identification. The technique enables recursive computations of estimates to be carried out. The typicalalgorithm forEF RLS is

$$
\begin{gathered}
\hat{\boldsymbol{\theta}}(t)=\hat{\boldsymbol{\theta}}(t-1)+P(t) \boldsymbol{\varphi}(t)\left[y(t)-\boldsymbol{\varphi}^{T}(t) \hat{\boldsymbol{\theta}}(t-1)\right], \\
R(t)=F(t) R(t-1)+\boldsymbol{\varphi}(t) \boldsymbol{\varphi}^{T}(t), \\
F=\mu I
\end{gathered}
$$

where $\widehat{\boldsymbol{\theta}}(t) \in R^{n}$ is the estimates of the parameter vector at time step $t, \boldsymbol{\varphi}(t) \in R^{n}$ is the observer data vector, $y(t) \in$ $R$ is the system output vector, $P(t) \in R^{n \times n}$ is the covariance matrix, $R(t) \in R^{n \times n}$ is the information matrix that is inverse of the covariance matrix, $F(t) \in R^{n \times n}$ is the forgetting matrix, $\mu \in(0,1)$ is the scalar forgetting factor.

The identification problem is stated as follows:

$$
\boldsymbol{\varsigma} \cong \boldsymbol{A} \widehat{\boldsymbol{\theta}},
$$

where the response variable vector is the following

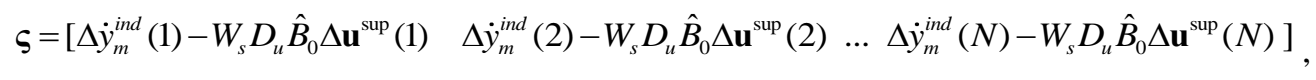

$\Delta \dot{y}_{m}^{\text {ind }}(1) \Delta \dot{y}_{m}^{\text {ind }}(2) \ldots \Delta \dot{y}_{m}^{\text {ind }}(N)$ is the record of derivative increment for $m$ component of the dynamic state vector $\boldsymbol{y}$, the predictor variable vector is based on the incremental signal for the control surface under study

$$
\mathbf{A}=\left[\Delta \mathrm{u}^{\text {ind }}(1) \Delta \mathrm{u}^{\text {ind }}(2) \ldots \Delta \mathrm{u}^{\text {ind }}(N)\right]^{T},
$$

$D_{u}$ is the allocation matrix, $W_{s}$ is the amplification matrix required to produce the supporting control signal $\boldsymbol{u}^{\text {sup }}$. Elements of $W_{s}$ specify how the individual actuator signals differ from the generic one. The terms $-W_{s} D_{u} \widehat{B}_{0} \boldsymbol{u}^{\text {sup }}(i)$, which are responsible for the subtraction of contribution from the supporting signal to the flight dynamics, are introduced in order to obtain the pure dynamics produced by the treated control surface.

\section{B. Tuning functions}

In the current section, we would like to design an adaptive augmentation to the baseline IBKS controller using the tuning function (TF) approach [17].

Here, we assume that actuator failure causes degradation of the actuation effectiveness. The dynamics of the general tracking error dynamics $\mathbf{z}_{g}=\left[\begin{array}{ll}\mathbf{z}_{\xi}^{T} & \mathbf{z}_{y}^{T}\end{array}\right]^{T} \in R^{7}$, which is measurable system state, is introduced with the following equations 


$$
\begin{aligned}
& \dot{\mathbf{z}}_{\xi}=\dot{\boldsymbol{\xi}}_{d}(t)-\dot{\boldsymbol{\xi}}_{0}\left(\mathbf{z}_{g}, t\right)-T_{\xi}\left(\boldsymbol{\omega}\left(\mathbf{z}_{g}, t\right)-\boldsymbol{\omega}_{0}\left(\mathbf{z}_{g}, t\right)\right), \\
& \dot{\mathbf{z}}_{y}=\dot{\mathbf{y}}_{d}\left(\boldsymbol{\omega}_{0}, t\right)-\dot{\mathbf{y}}\left(\mathbf{z}_{g}, t\right)-B_{0}(t)\left(\mathbf{u}\left(\mathbf{z}_{g}, t\right)-\mathbf{u}_{0}\left(\mathbf{z}_{g}, t\right)\right)-\dot{\chi}(\mathbf{u}, t),
\end{aligned}
$$

where $\xi_{d}, \xi_{0}, \dot{\boldsymbol{y}}, \dot{\boldsymbol{y}}_{d}$ are essentially locally bounded, uniformly in $t$ functions, $B_{0}(t)$ is the effectiveness matrix, an unknown, linear-parameterizable, essentially locally bounded function, $\dot{\chi}$ is influence of the CF, which is essentially locally bounded function, $\boldsymbol{u}$ is the baseline control input. $\widehat{B}_{0}: \mathbb{R}^{4 \times 14} \times[0, \infty) \rightarrow \mathbb{R}^{4 \times 14}$ is the estimate of $B_{0}$. We assume that there exists an unknown parameter vector $\widehat{\boldsymbol{\theta}} \in \mathbb{R}^{k}$ to be estimated such that $j$-column $\widehat{\boldsymbol{b}}_{j} \in \mathbb{R}^{k}$ of $\widehat{B}_{0}^{T}$ can be represented as

$$
\hat{\mathbf{b}}_{j}=\Phi_{j}^{T}\left(\boldsymbol{\xi}_{0}, \mathbf{y}_{0}, \mathbf{u}_{0}, t\right) \hat{\boldsymbol{\theta}},
$$

where $\Phi_{j}^{T}\left(\xi_{0}, \boldsymbol{y}_{0}, \boldsymbol{u}_{0}, t\right): \mathbb{R}^{3 \times 4 \times 14} \times[0, \infty) \rightarrow \mathbb{R}^{4 \times k}$ is the regressor function.

The estimation error is

$$
\widetilde{B}_{0}=B_{0}-\widehat{B_{0}} .
$$

In this case, the parameter estimation errors and its derivative are the following

$$
\widetilde{\boldsymbol{\theta}}=\boldsymbol{\theta}-\widehat{\boldsymbol{\theta}}, \quad \dot{\widetilde{\boldsymbol{\theta}}}=-\dot{\hat{\boldsymbol{\theta}}}
$$

For such a system, Lyapunov-based estimation algorithm can be designed

$$
\dot{\boldsymbol{\theta}}=-\Gamma \Phi_{j}^{T}\left(\boldsymbol{x}_{0}, \boldsymbol{u}_{0}\right) \overline{\mathbf{z}}_{y} \Delta \boldsymbol{u}_{j},
$$

where $\Gamma \in \mathbb{R}^{+}$are positive adaptation gains, $\Delta \boldsymbol{u}_{j}$ is $j$ th element of $\Delta \boldsymbol{u}$. Proof of stability could be found in [24].

\section{Simulation Results}

In this section, a simulation study of the ability of the discussed algorithms to tackle the failures is considered. A nonlinear model of the Boeing 747 aircraft, courteously provided by the consortium partner TU Munich, is used to validate the designed approach. This model is a variant of the GARTEUR RECOVER benchmark simulator [7]. The Boeing 747 is a large, transport aircraft with four wing-mounted engines. It has a length of approximately 70 meters, wingspan of 60 meters, and the maximum take-off weight is greater than 300 tons. The actuation of the Boeing 747 simulator corresponds to four ailerons, four elevators, two rudders, and four engines.

The nominal condition from which the simulation starts is a straight flight towards North with 340 knot of True Airspeed (TAS) and at an altitude of $5000 \mathrm{ft}$. The flight is developed under a low turbulence condition defined by a 20 -feet wind of $15 \mathrm{~m} / \mathrm{s}$ in North direction and a turbulence intensity exceedance probability of 0.01 . In the current research, a longitudinal motion is considered.

The algorithms are validated in the longitudinal motion of the aircraft. It is assumed that uncertainties are in the effectiveness of the elevator to control pitch. Three different scenarios were considered. In the first scenario, a control efficiency of the one of the elevators was reduced by half; in the second and third scenarios, nonlinear dynamics of the first and second order in one of the actuators, correspondingly, was added. Further explanation is provided bellow. For the GP identification, we had $x_{i}=\frac{V_{i}}{V_{\text {norm }}}$, where the normalizing constant $V_{\text {norm }}=345$ knot guarantees that the corresponding RBF centres are close to the unity. To have a proper comparison with EF RLS, we had the similar selection of the output data, namely, $\varsigma_{i}=\Delta \dot{y}_{i}^{\text {ind }}-W_{s} D_{u} \widehat{B}_{0} \Delta \boldsymbol{u}_{i}$. The maximum number $d$ of the $B V$ set was $3, \epsilon_{t o l}=$ $1 e-4, \epsilon_{u_{t o l}}=1 e-4, \sigma_{0}^{2}=5 e-9$. To obtain the appropriate overlapping between neighbouring kernels the RBF width is specified as follows 


$$
\sigma=\frac{0.2}{4(\sqrt{d}-1)^{2}}
$$

Forgetting function of EF RLS was $F=0.9999$. Adaptation gain for the TF update law was $\Gamma=150$.

\section{A. Two Failures and Loss of Effectiveness}

In the current section an ability of the developed controller is evaluated in a ca se of two failures (stuck-in-position) and $50 \%$ loss of effectiveness of the third elevator.

The results are presented in Fig.2. Here it is assumed that two elevators failed before $t=0 \mathrm{~s}$. For the adaptive algorithms, it is considered that these two failures detected and isolated also before $t=0 \mathrm{~s}$, which means that corresponding coefficients equal to zero in the $\widehat{B}_{0}$. The loss of effectiveness of one the two rest elevators simulated at $t=5 \mathrm{~s}$.
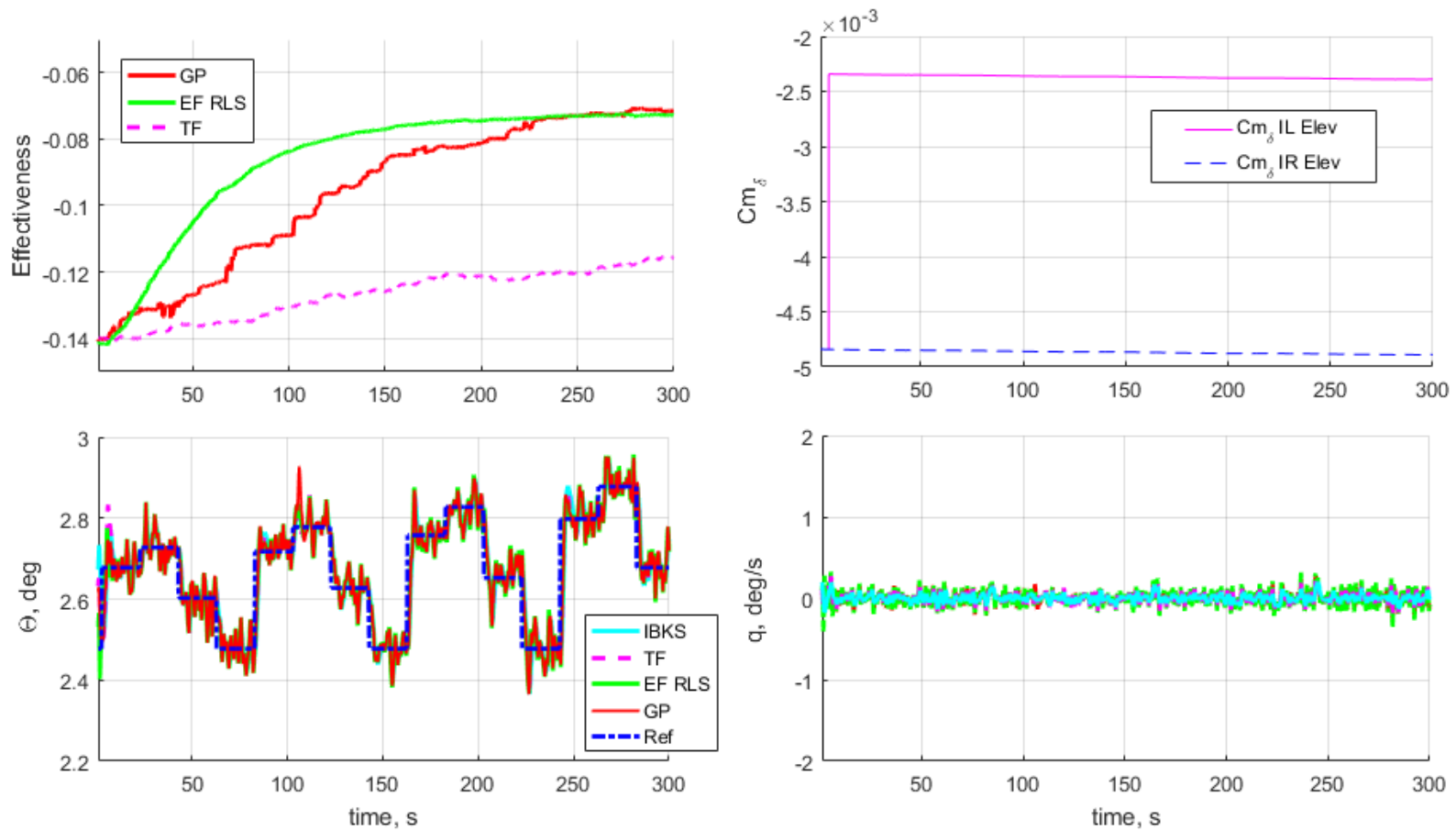

Fig.2 Two failures and loss of effectiveness

On the top left subplot, estimation of the failed elevator effectiveness obtained with GP is demonstrated. One can see that the identification is finished within $230 \mathrm{~s}$ after the "failure". At the same subplot, estimations provided by EF RLS and TF are also added for comparison purposes. One can see that performance of the GP is pretty similar to EF RLS, however, TF is much slower. It should be mentioned, that the slow estimation rate exhibited by TF is due to low adaptation gain and relatively small identification steps. They might be increased to improve the estimation rate, however, further increase of rate/amplitude caused loss of stability by the algorithm in other test cases. On the left bottom subplot, the pitch angle $\theta$ is presented, while in the bottom right figure the pitch rate $q$ is presented. On the right-top subplot the real effectiveness of two working elevators, namely, inner and outer elevators, are demonstrated. One can see that at $t=5 \mathrm{~s}$ the effectiveness of the inner elevator degraded (as assumed by the scenario). Effectiveness of the elevators failed before $t=0 \mathrm{~s}$ is zero.

From the obtained results one can conclude that the pure IBKS is quite robust to such type of failures, especially, when actuation redundancy is a vailable. When the mismatch between the real and model control efficiency is constant, i.e. when the system input affine property is conserved, the IBKS is able to cancel the produced uncertainty [22,26,43]. Nevertheless, the developed on-line estimations of control effectiveness could improve the control quality. This example shows that the proposed on-line GP identification provides good tracking of the variation of the control effectiveness. 


\section{B. First order dynamics}

Following the methodology proposed in [22], we also test the ability of the algorithms to counteract the uncertainties when the system input affine property was broken. This section considers the presence of the first order unmodeled actuator dynamics.

High level of redundancy (four elevators) allows conserving the input affine property for Boeing 747 even for the case of appearance of unmodeled dynamics in one of the actuators. To simulate the conditions where the input affine property is not valid anymore, we assume here that two elevators failed before $t=0 \mathrm{~s}$. For the adaptive algorithm, similar to the previous scenario, it is considered that two elevatorfailures were detected and isolated before $t=0 \mathrm{~s}$ and those corresponding coefficients equal to zero in $\widehat{B}_{0}$. Meanwhile, for the pure IBKS, it is considered, that the algorithm does not have access to new information about the control effectiveness, and thus, uses initial matrix $B_{0}$. At $t=150 \mathrm{~s}$, the nonlinear unmodeled dynamics arises at one of the two working actuators as a result of a failure. It was reported that many known actuatorfailures can be simulated with the first or second order actuatordynamics $[45,46]$. For the current scenario, we assumed the first order dynamics, represented with the following equation

$$
F(s)=(2 s+1)^{-1} .
$$

Comparison of behaviours of the GP-adaptive IBKS with other IBKS modification are presented in Fig.3.
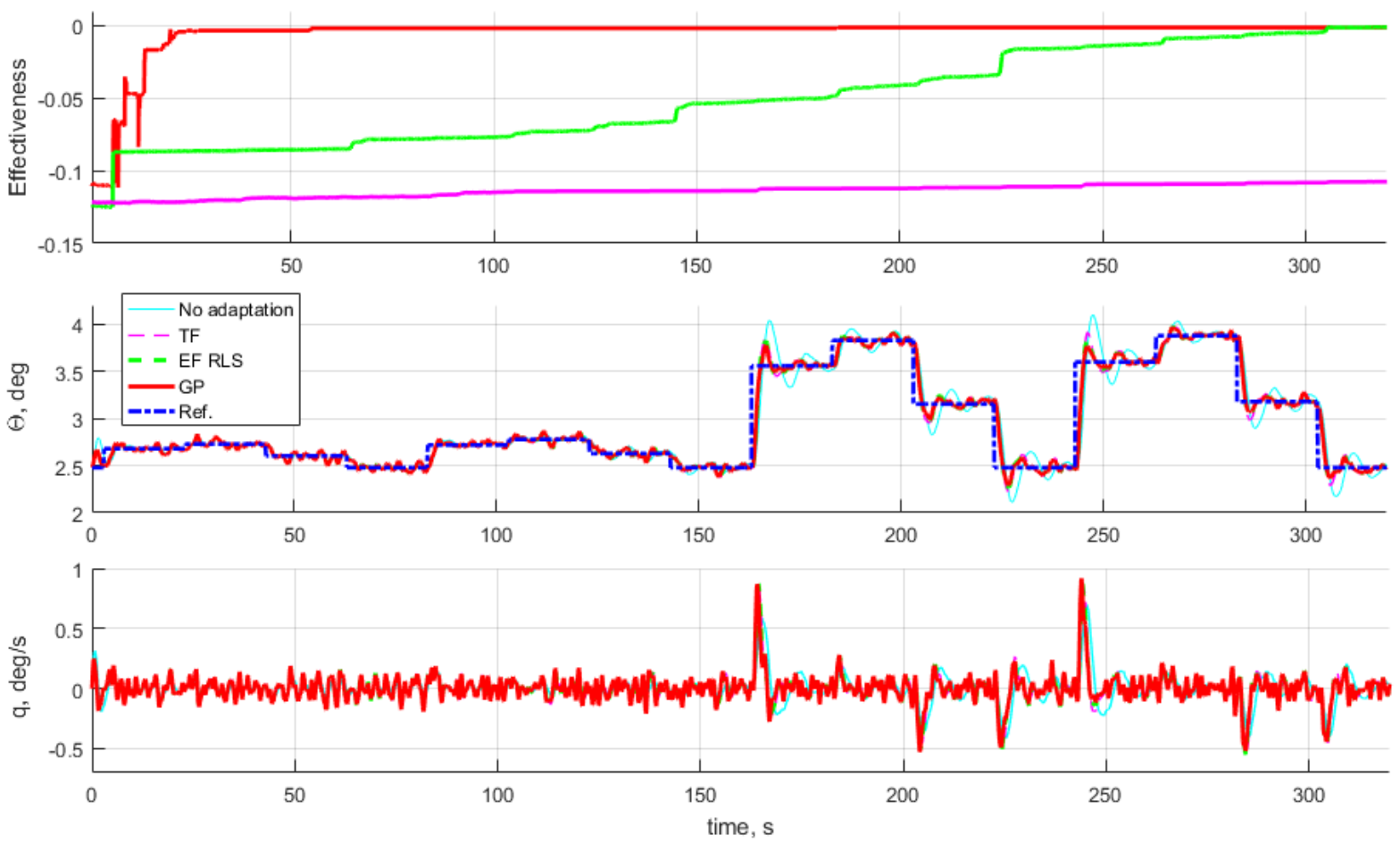

Fig.3 1-st order nonlinear dynamics

The top-level subplot demonstrates identification of the control effectiveness implemented with GP, EF RLS and TF techniques. The middle and bottom subplots show the parameters of the state vector $\theta$ and $q$. The first order actuator dynamics arose at $t=5 \mathrm{~s}$ has a significant effect on the performance of the IBKS algorithm, na mely, weakly damped oscillations are observed. Before $t=150 \mathrm{~s}$, the IBKS demonstrates robustness to failures for small demanded steps, namely, even with two failed elevators it follows the reference signal. At the same time, when at the moderate demanded pitch steps one can observe weakly damped oscillations in $\theta$ and $q$. These oscillations are much heavily dumped when any of the adaptation augmentation is applied.

The figure manifests that the on-line GP identification is significantly faster than other methods that leads to reduced overshoots in pitch as compared to other methods and, especially, to pure IBKS. GP is capable of relatively fast adaptation as compared to other considered algorithms even in ca se of small noise-to-signal ratio at $t<150 \mathrm{~s}$. 


\section{Second order dynamics}

Degradation of the IBKS performance due to loss of the input affine property because of presence of uncertainty in the form of non-linear dynamics in actuators is shown in the previous section. In this simulation experiment, the condition is examined further, namely, presence of non-linear dyna mics of the second order in one of the actuators is presented. Similar to the previous test case, we also assume that two elevators failed before $t=0 \mathrm{~s}$; for the adaptive controller, it is considered that these two failures detected and isolated before $t=0 \mathrm{~s}$ and those corresponding coefficients equal to zero in $\widehat{B}_{0}$. At $t=150 \mathrm{~s}$ at the one of the rest actuators the 2 -nd order unmodeled dynamics arises as a result of failure:

$$
F(s)=\left(2 s^{2}+s+1\right)^{-1} .
$$

Shown in Fig.4 is comparison between behaviour of IBKS and Adaptive IBKS in the considered scenario.
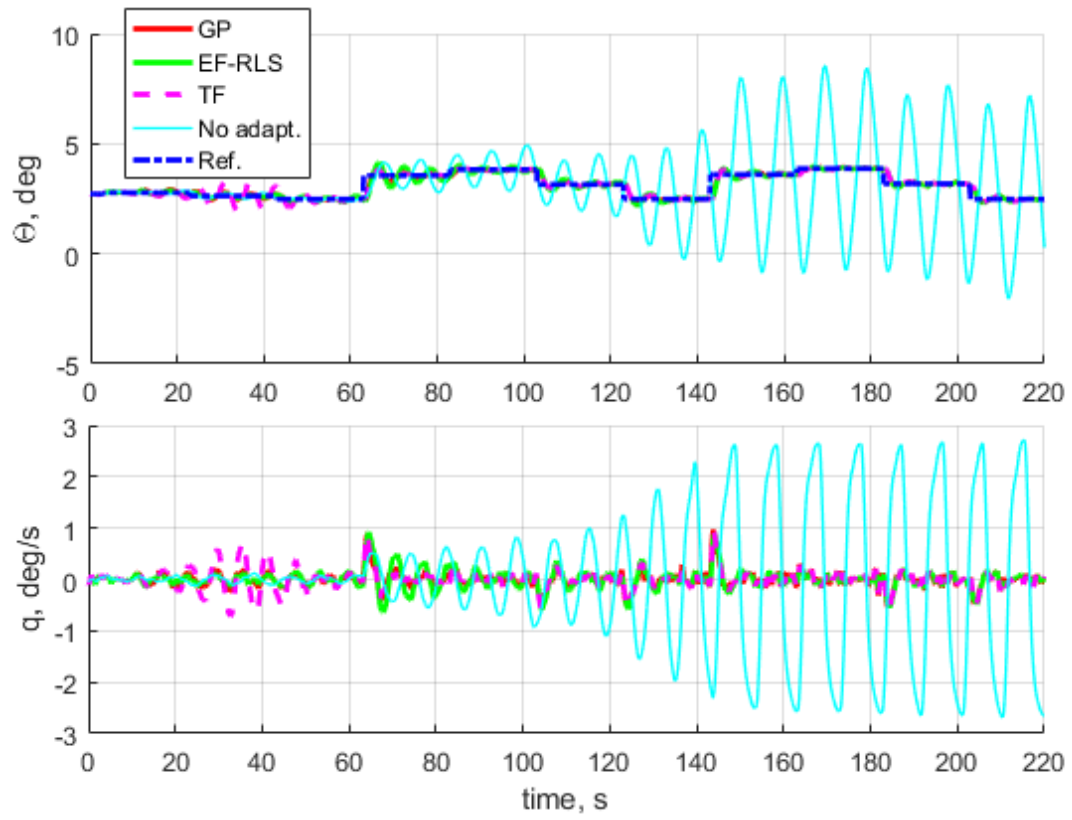

Fig.4 2-nd order nonlinear dynamics

Similar to the previous figures, the transition processes of state vector $\theta$ and $q$ are demonstrated. From the figure, one can see that under the presence of 2-nd order dynamics the IBKS control suffers from the instability in the form of high-a mplitude limit-cycle oscillations. Such a nonlinear dynamics is caused by interaction between failed and nonfailed eleva tors. Meantime, a daptively augmented IBKS manifests the system stability and good tracking performance.

Three different adaptive strategies are scrutinized in Fig.5. All three algorithms provide stability. Similar to the previous scenario, GP demonstrates the fastest adaptation rate and thus the best tracking performance among considered methods because it "switch off" harmful interaction of the failed and non-failed elevator. 

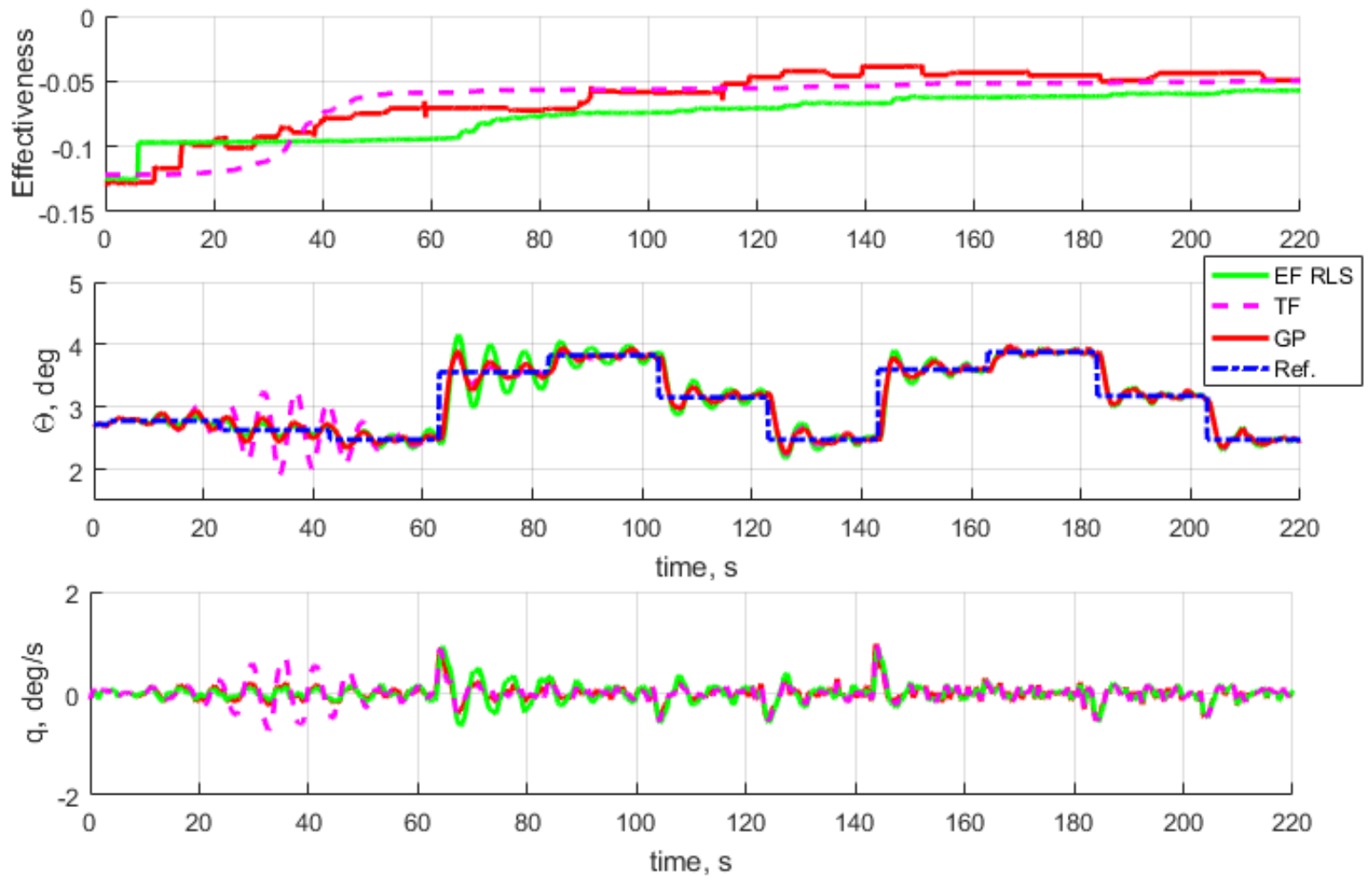

Fig.5 Comparison of different estimators: 2-nd order nonlinear dynamics

\section{Conclusion}

IncrementalBackstepping is recently developed technique with a reduced dependency on on-board aircraft model. This approach uses estimates of the state derivatives and the current actuator states to linearize the flight dynamics with respect to current state. Our results and results of the other researchers revea led robustness of the IBKS to actuator failures when the system remains input affine, even for the case of multiple failures. However, as we have shown in the current study, in severe conditions, with a combination of multiple failures and presence of unmodelle $\mathrm{d}$ actuator dynamics, the system dynamics might loss its input affine property. Such conditions might be a case for not only scenarios considered in the current study but also for some others, for example, in case of partialloss of effectiveness and transport delays. As a result, the stability of the system cannot be guaranteed anymore and adaptive augmentation is required to compensate the unmodelled dynamics.

In this research, we proposed GP based adaptive augmentation to IBKS, which uses budgeted sparse GP algorithm for on-line identification of control effectiveness. GP is an elegant and efficient non-parametric estimation procedure developed within the Bayesian paradigm. To make Bayesian interference trackable on -line, the posterior is projected to the closest Gaussian process by a single sequential sweep through the examples. Within the approach, the number of basis vectors is limited with predefined "budget" to make the algorithm computationally efficient. The input vectors providing the maximum information richness to the basis set, namely, having maximum scores, are selected as the basis vectors, while basis vectors with lowest scores are deleted from the set.

Performance of the GP-adapted IBKS was studied in simulations of three different failure scenarios developed for Boeing 747 involving multiple failures with partial loss of effectiveness, unmodelled actuator dynamics of the first and the second orders. Our results manifested improved stability and tracking performance characteristics of the IBKS controller with control efficiency estimations as compared to the baseline IBKS. More precise information fed to the baseline controller by the estimator improved tracking performance for the case of loss of effectiveness, cancelled undesired oscillations observed for the IBKS in case of first order actuator dynamics and prevented from a loss of stability for the second-order actuator dynamics. Performance of the adaptive IBKS based on the GP estimator is evaluated by comparison with EF RLS and TF. For the partial loss of efficiency, GP and EF RLS demonstrated pretty similar results, however, for the cases of unknown nonlinear actuator dynamics, GP showed faster adaptation leading to improved tracking performance. It is well known that EF RLS works well, however it shows less efficiency for 
time varying process. TF algorithm demonstrated slower adaptation as a result of small adaptation gain, higher adaptation gains leaded to loss of stability. Additional top-level algorithms, switching-on and switching-off TFestimations might be applied to make possible higher adaptation gains. However, such additional structures might make the overall control algorithm more complex. We could conclude that GP based adaptation loop augmenting IBKS provides the best overall result among considered methods.

\section{Acknowledgments}

This research is partially funded by the European Union in the scope of INCEPTION project, which has received funding from the EU's Horizon2020 Research and Innovation Programme under grant agreement No. 723515.

\section{References}

[1] EASA. European Aviation Safety Agency. Annual Safety Review 2014. 2014.

[2] Abramov, N. B., Goman, M. G., Khrabrov, A. N., and Soemarwoto, B. I. "Aerodynamic Modeling for Poststall Flight Simulation of a Transport Airplane.” Journal of Aircraft, Vol. 56, No. 4, 2019, pp. 1427-1440. https://doi.org/10.2514/1.c034790.

[3] Murch, A. M., and Foster, J. V. "Recent NASA Research on Aerodynamic Modeling of Post-Stall and Spin Dynamics of Large Transport Airplanes." Collection of Technical Papers - 45th AIAA Aerospace Sciences Meeting, Vol. 8, No. January, 2007, pp. 5553-5572. https://doi.org/10.2514/6.2007-463.

[4] Ignatyev, D. I., Sidoryuk, M. E., Kolinko, K. A., and Khrabrov, A. N. "Dynamic Rig for Validation of Control Algorithms at High Angles of Attack.” Journal of Aircraft, Vol. 54, No. 5, 2017, pp. 1760-1771. https://doi.org/10.2514/1.C034167.

[5] Ignatyev, D., and Khrabrov, A. "Experimental Study and Neural Network Modeling of Aerodynamic Characteristics of Canard Aircraft at High Angles of Attack." Aerospace, Vol. 5, No. 1, 2018, p. 26. https://doi.org/10.3390/aerospace5010026.

[6] Goman, M. G., Khramtsovsky, A. V., and Kolesnikov, E. N. "Evaluation of Aircraft Performance and Maneuverability by Computation of Attainable Equilibrium Sets." Journal of Guidance, Control, and Dynamics, Vol. 31, No. 2, 2008 , pp. 329-339. https://doi.org/10.2514/1.29336.

[7] Smaili, H., Jan, B., Lombaerts, T., and Stroosma, O. “A Benchmark for Fault Tolerant Flight Control Evaluation.” IFAC Proceedings Volumes (IFAC-PapersOnline), No. December, 2009, pp. 241-246. https://doi.org/10.3182/20090630-4-ES2003.0246.

[8] Ignatyev, D. I., and Khrabrov, A. N. "Neural Network Modeling of Unsteady Aerodynamic Characteristics at High Angles of Attack.” Aerospace Science and Technology, Vol. 41, 2015, pp. 106-115. https://doi.org/10.1016/j.ast.2014.12.017.

[9] Yucelen, T., and Calise, A. J. "Derivative-Free Model Reference Adaptive Control." Journal of Guidance, Control, and Dynamics, Vol. 34, No. 4, 2012, pp. 933-950. https://doi.org/10.2514/1.53234.

[10] Hovakimyan, N., and Cao, C. L1 Adaptive Control Theory: Guaranteed Robustness with Fast Adaptation. 2010.

[11] Falconi, G. P., Heise, C. D., and Holzapfel, F. "Novel Stability Analysis of Direct MRAC with Redundant Inputs." 24th Mediterranean Conference on Control and Automation, MED 2016, 2016, pp. $176-181$. https://doi.org/10.1109/MED.2016.7536060.

[12] Chu, Q. P., Joosten, D. A., Smaili, M. H., Stroosma, O., J. Lombaerts, T. J., and Mulder, J. A. "Piloted Simulator Evaluation Results of New Fault-Tolerant Flight Control Algorithm.” Journal of Guidance, Control, and Dynamics, Vol. 32, No. 6, 2009, pp. 1747-1765. https://doi.org/10.2514/1.44280.

[13] Blakelock, J. H. Automatic Control of Aircraft and Missiles. Wiley, New York, 1991.

[14] Shue, S. P., Sawan, M. E., and Rokhsaz, K. “Mixed H/H8 Method Suitable for Gain Scheduled Aircraft Control.” Journal of Guidance, Control, and Dynamics, Vol. 20, No. 4, 1997, pp. 699-706.

[15] Slotine, J.-J.E., and Li, W. Applied Nonlinear Control. 1991.

[16] Antonios Tsourdos, and Brian White. "Adaptive Flight Control Design for Nonlinear Missile." Control Engineering Practice, Vol. 13, No. 3, 2005, pp. 373-382.

[17] Krstic, M., Kokotovic, P. V., and Kanellakopoulos, I. Nonlinear and Adaptive Control Design. John Wiley \& Sons, Inc., USA., 1995.

[18] Lee, C.-H., Kim, T.-H., and Tahk, M.-J. “Agile Missile Autopilot Design Using Nonlinear Backstepping Control with Time-Delay Adaptation.” Trans. Japan Soc. Aero. Space Sci., Vol. 57, No. 1, 2014, pp. 9-20.

[19] Azinheira, J. R., Moutinho, A., and De Paiva, E. C. "Airship Hover Stabilization Using a Backstepping Control Approach." Journal of Guidance, Control, and Dynamics, Vol. 29, No. 4, 2006, pp. 903-914. https://doi.org/10.2514/1.17334.

[20] Sun, L. G., de Visser, C. C., Chu, Q. P., and Falkena, W. "Hybrid Sensor-Based Backstepping Control Approach with Its Application to Fault-Tolerant Flight Control." Journal of Guidance, Control, and Dynamics, Vol. 37, No. 1, 2013, pp. 5971. https://doi.org/10.2514/1.61890.

[21] Sieberling, S., Chu, Q. P., and Mulder, J. A. "Robust Flight Control Using Incremental Nonlinear Dynamic Inversion and Angular Acceleration Prediction.” Journal of Guidance, Control, and Dynamics, Vol. 33, No. 6, 2010, pp. $1732-1742$. https://doi.org/10.2514/1.49978.

[22] Ignatyev, D. I., Shin, H. S., and Tsourdos, A. "Two-Layer Adaptive Augmentation for Incremental Backstepping Flight Control of Transport Aircraft in Uncertain Conditions.” Aerospace Science and Technology, Vol. 105, 2020, p. 106051. 
Bhardwaj, P., Akkinapalli, V. S., Zhang, J., Saboo, S., and Holzapfel, F. "Adaptive Augmentation of Incremental Nonlinear Dynamic Inversion Controller for an Extended F-16 Model.” AIAA Scitech 2019 Forum, No. January, 2019, pp. 1-21. https://doi.org/10.2514/6.2019-1923.

[24] van Gils, P., Van Kampen, E.-J., de Visser, C. C., and Chu, Q. P. “Adaptive Incremental Backstepping Flight Control for a High-Performance Aircraft with Uncertainties." AIAA Guidance, Navigation, and Control Conference, No. January, 2016. https://doi.org/10.2514/6.2016-1380.

[25] Falconi, G. P., Marvakov, V. A., and Holzapfel, F. "Fault Tolerant Control for a Hexarotor System Using Incremental Backstepping." 2016 IEEE Conference on Control Applications, CCA 2016, No. 1, 2016, pp. $237-242$. https://doi.org/10.1109/CCA.2016.7587842.

[26] Jeon, B.-J., Seo, M.-G., Shin, H.-S., and Tsourdos, A. "Understandings of Classical and Incremental Backstepping Controllers with Model Uncertainties." IEEE Transactions on Aerospace and Electronic Systems, Vol. 9251, No. c, 2019, pp. 1-1. https://doi.org/10.1109/taes.2019.2952631.

[27] Akkinapalli, V. S., Falconi, G. P., and Holzapfel, F. "Fault Tolerant Incremental Attitude Control Using Online Parameter Estimation for a Multicopter System." 2017 25th Mediterranean Conference on Control and Automation, MED 2017, 2017, pp. 454-460. https://doi.org/10.1109/MED.2017.7984159.

[28] Ignatyev, D. I., Shin, H. S., and Tsourdos, A. Two-Layer on-Line Parameter Estimation for Adaptive Incremental Backstepping Flight Control for a Transport Aircraft in Uncertain Conditions. No. 52, 2019, pp. 411-416.

[29] Ignatyev, D., Shin, H.-S., and Tsourdos, A. Two-Layer Fault Detection for Incremental Flight Control of Fixed-Wing UAV. 2019.

[30] Ignatyev, D. I., Shin, H.-S., and Tsourdos, A. "Fault Detection, Isolation and Adaptive Augmentation for Incremental Backstepping Flight Control.” IFAC-PapersOnLine, Vol. 53, No. 2, 2020, pp. 14799-14804. https://doi.org/10.1016/j.ifacol.2020.12.1912.

[31] Karl Johan Åström, B. W. Adaptive Control. Addison-Wesley Longman Publishing Co., Inc., Boston, MA, USA, 1994.

[32] Klein, V., and Morelli, E. Aircraft System Identification - Theory and Practice. AIAA, Inc., Blacksburg, USA, 2006.

[33] Calise, A., Shin, Y., and Johnson, M. "A Comparison Study of Classical and Neural Network Based Adaptive Control of Wing Rock.” No. August, 2004, pp. 1-17. https://doi.org/10.2514/6.2004-5320.

[34] Ignatyev, D. I., Shin, H. S., and Tsourdos, A. "Bayesian Calibration for Multiple Source Regression Model." Neurocomputing, Vol. 318, 2018, pp. 55-64. https://doi.org/10.1016/j.neucom.2018.08.027.

[35] Boëly, N., and Botez, R. M. New Approach for the Identification and Validation of a Nonlinear F/A-18 Model by Use of Neural Networks. IEEE Transactions on Neural Networks. 11. Volume 21, 1759-1765.

[36] Narendra, K. S. "Neural Networks for Control: Theory and Practice." Proceedings of the IEEE, Vol. 84, No. 10, 1996, pp. 1385-1406. https://doi.org/10.1109/5.537106.

[37] Csató, L., and Opper, M. "Sparse On-Line Gaussian Processes.” Neural Computation, Vol. 14, No. 3, 2002 , pp. 641-668. https://doi.org/10.1162/089976602317250933.

[38] Ioannou, P. A., and Kokotovic, P. V. "Instability Analysis and Improvement of Robustness of Adaptive Control." Automatica, Vol. 20, No. 5, 1984, pp. 583-594. https://doi.org/https://doi.org/10.1016/0005-1098(84)90009-8.

[39] Chowdhary, G., Kingravi, H. A., How, J. P., and Vela, P. A. "Bayesian Nonparametric Adaptive Control Using Gaussian Processes.” IEEE TRANSACTIONS ON NEURAL NETWORKS AND LEARNING SYSTEMS, Vol. 26, No. 3, 2015, pp. 537-550. https://doi.org/10.1109/TNNLS.2014.2319052.

[40] Grande, R. C., Chowdhary, G., and How, J. P. "Experimental Validation of Bayesian Nonparametric Adaptive Control Using Gaussian Processes." Journal of Aerospace Information Systems, Vol. 11, No. 9, 2014, pp. 565-578. https://doi.org/10.2514/1.i010190.

[41] Https://Inception-H2020.Tekever.Com/.

[42] Cordeiro, R. A., Azinheira, R., and Moutinho, A. Cascaded Incremental Backstepping Controller for the Attitude Tracking of Fixed-Wing Aircraft. 2019.

[43] Cordeiro, R. A., Azinheira, J. R., and Moutinho, A. "Addressing Actuation Redundancies in Incremental Controllers for Attitude Tracking of Fixed-Wing Aircraft.” IFAC-PapersOnLine, Vol. 52, No. 12, 2019, pp. $417-422$. https://doi.org/10.1016/j.ifacol.2019.11.279.

[44] Vapnik, V. N. The Nature of Statistical Learning Theory. Springer, 1999.

[45] Bošković, J. D., and Mehra, R. K. "Failure Detection, Identification and Reconfiguration System for a Redundant Actuator Assembly." IFAC Proceedings Volumes (IFAC-PapersOnline), Vol. 36, No. 5, 2003, pp. $411-416$. https://doi.org/10.1016/S1474-6670(17)36526-6.

[46] Bošković, J. D., Bergstrom, S. E., and Mehra, R. K. "Adaptive Accommodation of Failures in Second-Order Flight Control Actuators with Measurable Rates." Proceedings of the American Control Conference, Vol. 2, No. July, 2005, pp. 10331038. https://doi.org/10.1109/acc.2005.1470096. 
$2021-12-29$

\section{Gaussian process adaptive incremental backstepping flight control}

Ignatyev, Dmitry I.

AIAA

Ignatyev D, Shin H-S, Tsourdos A. (2021) Gaussian process adaptive incremental backstepping flight control. In: AIAA SciTech 2022 Forum, 3-7 January 2022, San Diego, CA, USA and Virtual Event https://doi.org/10.2514/6.2022-2032

Downloaded from Cranfield Library Services E-Repository 